The problem of Anisotropy in Electrical Impedance Tomography

Breckon, William R.

1992

MIMS EPrint: 2008.44

Manchester Institute for Mathematical Sciences

School of Mathematics

The University of Manchester

\footnotetext{
Reports available from: http://eprints.maths.manchester.ac.uk/

And by contacting: The MIMS Secretary

School of Mathematics

The University of Manchester

Manchester, M13 9PL, UK
} 


\title{
The Problem of Anisotropy in Electrical Impedance Tomography
}

\author{
William Breckon \\ EIT Research Group, School of Computing and Mathematical Sciences, \\ Oxford Polytechnic, Oxford, OX3 OBP, U.K.
}

\begin{abstract}
The reconstruction algorithms used for Electrical Impedance Tomography assume that the conductivity is isotropic. If the conductivity is anisotropic then the solution to the reconstruction problem is not unique. As many body tissues are anisotropic this presents a problem. Recent mathematical results suggest that anisotropic conductivities which are indistinguishable using EIT differ by a distortion of the domain. In addition, those anisotropic distributions consistent with some anisotropic distribution are unlikely to occur in practice. What is need for the success of EIT in the anisotropic case is sufficient additional knowledge of the structure of the anisotropy to make the solution of the reconstruction problem unique.
\end{abstract}

\section{INTRODUCTION}

The electrical conductivity of the human body has anisotropic regions. The most striking case of this is skeletal muscle for which the longitudinal conductivity has been found to be 15 times the transverse [1]. Blood becomes more conductive in the direction of its velocity as its flow rate increases. Here factors of up to 1.2 reported [2]. In Electrical Impedance Tomography (EIT) the conductivity is sought by surface measurements of current and voltage. All EIT reconstruction algorithms used in practice assume that the conductivity is isotropic.

\section{ANISOTROPY AND UNIQUENESS}

In EIT the data collected are pairs $(j, v)$ of surface current density $\mathrm{j}$ and surface voltage $v$ sampled at specific electrodes. This gives us a necessarily incomplete knowledge of the transfer impedance operator $\mathrm{R}$ where $\mathrm{Rj}=\mathrm{v}$. In the interior of the body $\Omega$ (taken to be three dimensional), the potential $u$ satisfies

$$
\nabla \cdot \sigma \nabla \mathbf{u}=0
$$

On the boundary $\partial \Omega, v=u$ and $j=-\sigma \nabla u \cdot n$ where $\mathbf{n}$ is the outward normal to $\partial \Omega$.

Theoretically it is possible to recover an unknown isotropic conductivity $\sigma$ from a complete knowledge of $R(\sigma)$ [3], in the sense that different conductivities give rise to different transfer impedance operators. However for an anisotropic conductivity this is not the case.

An example of two anisotropic conductivities indistinguishable by EIT is due to Luc Tartar [4]. Take an invertible smooth mapping (diffeomorphism) $\quad \mathrm{f}: \Omega \rightarrow \Omega$ which is the identity on $\partial \Omega$ and whose derivative $\operatorname{Df}(x)=\mathrm{I}$, the identity matrix, for $x \in \partial \Omega$. For any conductivity tensor $\sigma=\left(\sigma_{\mathrm{ij}}\right)$ define another conductivity $\tau=\left(\tau_{\mathrm{ij}}\right)$ by

$$
\tau(f(x))=|\operatorname{det}(\operatorname{Df}(x))|^{-1} \operatorname{Df}(x)^{t} \sigma \operatorname{Df}(x) .
$$

If $\nabla \cdot \sigma \nabla \mathrm{u}=0$ then $\nabla \cdot \tau \nabla \mathrm{w}=0$ where $\mathrm{w}(\mathrm{x})=\mathrm{u}(\mathrm{f}(\mathrm{x}))$ and the boundary voltage data will agree $u=w$ on $\partial \Omega$. Also the current densities will agree as $\sigma \nabla \mathbf{u}=\tau \nabla \mathbf{w}$ on $\partial \Omega$. Hence $R(\sigma)=R(\tau)$. The practical implication of this is that we may find an anisotropic conductivity which is consistent with our EIT data, but that any distortion of this (where the distortion does not affect the surface) would also be consistent. In [5] it is conjectured that these distorted conductivities are the only ones consistent with the complete EIT data, and this is proved under fairly strong conditions on $\sigma$.

\section{RIEMANNIAN GEOMETRY}

What happens if we ignore the anisotropy and attempt to find an isotropic conductivity consistent with the data $R(\sigma)$ from an anisotropic distribution? With complete data, this will only be possible (accepting the restrictions in [5]) if $\sigma$ is a distortion of an isotropic conductivity $\imath=\left(\phi \delta_{i j}\right)$ where $\phi$ is a positive scalar function. However there are good reasons to suppose that in practice this is unlikely. To explain this further some Riemannian Geometry is required. Following [5] we define a Riemann metric $\mathbf{g}$ in Euclidean coordinates on $\Omega$

$$
\left(g_{\mathrm{ij}}\right)=(\operatorname{det} \sigma) \sigma^{-1} .
$$

The Laplacian operator with respect to this metric is given by

$$
\Delta_{\mathbf{g}} u=\sum_{i, j=1}^{3}(\operatorname{det} \mathbf{g})^{-1 / 2} \frac{\partial}{\partial x^{i}}\left((\operatorname{det} \mathbf{g})^{1 / 2} g^{i j} \frac{\partial u}{\partial x^{j}}\right)
$$

and $\Delta_{\mathrm{g}} \mathrm{u}=0$ is equivalent to $\nabla \cdot \sigma \nabla \mathrm{u}=0$. Denoting the Neumann to Dirichlet mapping for Laplace's equation $\Delta_{g} u$ $=0$ by $R(\mathbf{g})$, we have $R(\mathbf{g})=R(\sigma)$. 
In the language of Riemannian geometry, a metric which in some coordinate system is $\left(\delta_{i j}\right)$ is called a flat metric. A metric equivalent via a diffeomorphism to some $\imath=\left(\phi \delta_{i j}\right)$ is called conformally flat. Consequently any conductivity $\sigma$ which is a distortion of an isotropic distribution must be associated with a conformally flat metric. For convenience we will call $\sigma$ a conformally flat conductivity.

In the case of a three dimensional domain, there is a simple test for conformally flat metrics. The Cotton tensor [6] is defined in terms of the Ricci tensor $R_{i j}$ and scalar curvature $\mathrm{R}$ of the metric. Using a comma to denote covariant differentiation it is given by

$$
C_{i j k}=R_{i j, k}-R_{i k, j}+\frac{1}{4}\left(R_{, k} g_{i j}-R_{, j} g_{i k}\right) .
$$

A metric is conformally flat if and only if its Cotton tensor is identically zero [7]. Ultimately, the Cotton tensor is a function of the $\sigma$ and its partial derivatives up to order 3 . Intuitively, the vanishing of 27 functions of the partial derivatives of $\sigma$ would not happen by chance. Mathematically this can be expressed by saying that in the space of three times continuously differentiable conductivity tensors $\sigma$, those which are not conformally flat form an open dense set. This follows from the Thom transversality theorem [8]. Being not conformally flat is an example of a generic property - in a particular sense, almost all conductivities have this property.

\section{STRUCTURED ANISOTROPY}

In practice the body does not consist of a randomly arranged anisotropic conductivity. The anisotropy of muscles is oriented with the muscle fibres, and these fibres are often arranged in sheets with a consistent orientation. Blood flows largely in well defined tubes, its anisotropy aligned with the flow vector. These physiological facts place specific geometrical constraints on $\sigma$. A distortion will move and distort these structures, but not eliminate them. Hence even in the presence of anisotropy, EIT may be able to indicate the presence or absence of particular features but not give position or specific quantitative information. However if sufficient additional information is included, such as the positions of certain anatomical 'land marks' it may be possible to eliminate some of the ambiguity.

\section{ACKNOWLEDGMENT}

The author thanks Professor Simon Donaldson for helpful discussions on differential geometry.

\section{REFERENCES}

[1] D.C. Barber and B.H. Brown, "Applied potential tomography", J. Phys. E. Sci. Instrum. 17, 1984, pp723-733.

[2] R. Lamberts., K.R. Visser, Zijlstra W.G., "Impedance Cardiography",1984, Van Gorcum , Assen.

[3] J. Sylvester and G. Uhlmann, "A global uniqueness theorem for an inverse boundary value problem", Ann Math., 125, 1987, pp153-169.

[4] R.V Kohn and M. Vogelius, "Identification of an unknown conductivity by means of measurements at the boundary", Proc. SIAM-AMS Symp. on Inverse Problems, New York, 1983, pp113-123

[5] J.M. Lee and G. Uhlmann, "Determining Anisotrpic Real-Analytic Conductivities by Boundary Measurements", Comm. Pure Appl. Math., vol 42, 1989, pp1097-1112.

[6] J.A. Schouten, "Über die konforme Abbildung $n$ dimensionaler Mannigfaltigkeiten mit quadratischer Maßbestimmung auf eine Mannigfaltigkeit mit euklidischer Maßbestimmung", Mathematische Zeitschrift, vol 11, 1921, pp58-88.

[7] L.P. Eisenhart, Riemannian Geometry, Princeton University Press, 1926.

[8] J. Palis and W. de Melo. Geometric Theory of Dynamical Systems, Springer-Verlag, New York, 1982. 